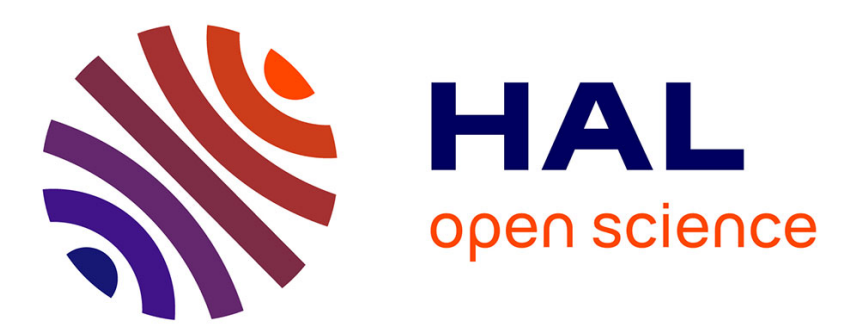

\title{
Inégalité de milieu d'origine et destin salarial en France,1977-1993
}

Arnaud Lefranc, Nicolas Pistolesi, Alain Trannoy

\section{To cite this version:}

Arnaud Lefranc, Nicolas Pistolesi, Alain Trannoy. Inégalité de milieu d'origine et destin salarial en France,1977-1993. Revue d'Economie Politique, 2007, 117 (1), 10.3917/redp.171.0091 . hal-01651117

\section{HAL Id: hal-01651117 https://hal.science/hal-01651117}

Submitted on 28 Nov 2017

HAL is a multi-disciplinary open access archive for the deposit and dissemination of scientific research documents, whether they are published or not. The documents may come from teaching and research institutions in France or abroad, or from public or private research centers.
L'archive ouverte pluridisciplinaire HAL, est destinée au dépôt et à la diffusion de documents scientifiques de niveau recherche, publiés ou non, émanant des établissements d'enseignement et de recherche français ou étrangers, des laboratoires publics ou privés. 


\title{
Inégalité de milieu d'origine et destin salarial en France, 1977-1993 *
}

\author{
Arnaud LEFRANC ${ }^{\dagger} \quad$ Nicolas PISTOLESI ${ }^{\ddagger} \quad$ Alain TRANNOY TR $^{\S}$
}

4 décembre 2006

\begin{abstract}
Résumé
Etudier l'inégalité des chances consiste à étudier des distributions concernant les résultats d'une génération conditionnellement à des caractéristiques du milieu familial. Nous étudions l'évolution de l'inégalité des chances entre 1977 et 1993 dans l'obtention du revenu salarial en fonction du rang ou du niveau de revenu du père à l'aide de l'enquête FQP. En faisant appel à des instruments de dominance stochastique, on montre que l'inégalité des chances est restée stable quand on conditionne par rapport au niveau du revenu du père mais qu'elle a diminué lorsque l'on conditionne par rapport au rang du père. On propose ensuite un indice d'inégalité des chances basé sur l'écart logarithmique moyen et une méthode originale de décomposition de l'évolution de cet indice qui s'appuie sur des régressions intergénérationnelles de revenu. Il est alors montré que le premier résultat s'explique par la constance de la valeur estimée de l'élasticité intergénérationnelle de revenu, tandis que le second résultat provient de la baisse de l'inégalité des salaires dans la génération précédente. En d'autres termes, l'égalisation partielle des revenus au sein de la génération des descendants, entre les deux dates, provient uniquement de l'égalisation des revenus au sein du groupe des pères, entre ces deux dates, et non pas d'un plus faible déterminisme social.
\end{abstract}

JEL Codes : D1, D3, J3

Mots clés : Inégalité des chances, dominance stochastique, circonstance, écart logarithmique moyen.

\footnotetext{
*Nous remercions Marc Fleurbaey et un rapporteur anonyme pour leurs remarques. Nous restons seuls responsables des éventuelles erreurs ou omissions.

${ }^{\dagger}$ Institut Universitaire Européen (Florence), THEMA, IDEP et Université de Cergy-Pontoise. arnaud. lefranc@u-cergy.fr

‡THEMA, Université de Cergy-Pontoise. nicolas.pistolesi@u-cergy.fr

$\S$ EHESS, GREQAM-IDEP. alain.trannoy@ehess.cnrs-mrs.fr
} 


\begin{abstract}
To analyze equality of opportunity we examine the influence of family characteristics on the distribution of outcome for a given generation. Here, we study changes in equality of opportunity for income acquisition between 1977 and 1993, conditional on father's income level and income rank, using the FQP surveys. First, resorting to stochastic dominance criteria, we show that inequality of opportunity appears stable over this period, if one conditions on father's income level, but has decreased, if one conditions on father's income rank. Second, we offer an equality of opportunity index, based on mean logarithmic deviation and show how to decompose the evolution over time of this index using standard intergenerational earnings regression analysis. Using this framework, we show that the first results arises from the stability over time in the intergenerational earnings elasticity while the second stems from a reduction in earnings inequality among fathers between 1977 and 1993. In other words, lower earnings inequality among parents, between these two dates, has translated into lower earnings inequality among children, although the strength of the association between the income of successive generations has remained unchanged.
\end{abstract}

Keywords : Inequality of opportunity, stochastic dominance, circumstances,mean logarithmic deviation. 


\section{Introduction}

L'analyse empirique des inégalités de revenu s'est longtemps focalisée sur la mesure des inégalités inter-individuelles de revenus ou de conditions de vie et sur l'étude de leurs évolutions dans le temps. Cette façon de procéder, compréhensible au regard des données généralement disponibles, n'est cependant pas totalement en phase avec les recommandations de certains philosophes de la responsabilité, tels que Dworkin (1981), Arneson (1989), Cohen (1989), ou encore Roemer (1998). Ces auteurs soulignent que certaines inégalités peuvent être moralement acceptables alors que d'autres ne le sont pas. Une politique redistributive devrait avant tout se focaliser sur la réduction de l'inégalité tenant aux différences d'environnement dans lequel les individus ont été placés depuis leur enfance. Ces différences d'environnement familial et social, les circonstances pour Roemer, présentent un caractère d'exogénéité et par là même ne sont pas éthiquement acceptables en tant que source d'inégalitế

Dans une perspective intergénérationnelle, le débat concerne d'abord l'influence du milieu parental sur le devenir des enfants. Roemer (2004) et Dardanoni et alii (2005) présentent quatre canaux de transmission à travers lesquels les parents affectent les capacités de revenu de leurs enfants : la transmission génétique des capacités, la formation des croyances et des aptitudes, la constitution des préférences et des aspirations, le partage des liens sociaux. Le revenu s'avère un puissant instrument permettant aux parents d'influencer le devenir de leurs enfants à travers les trois derniers canaux de transmission. Il est en même temps un indicateur de la réussite économique des parents qui est corrélé positivement à leur faculté génétique et donc à celle de leur progéniture. Le revenu des parents apparaît donc comme une mesure omnibus de la capacité des ascendants à influencer le devenir économique des descendants. En outre, comme il est difficile de tenir pour responsable un enfant du revenu de ses parents, il possède bien le caractère d'exogénéité qui permet de le qualifier de circonstance. Dans cette perspective, toute différence de revenu des descendants associée à des différences de revenu des parents sera interprétée comme un symptôme d'inégalité des chances. Cet article s'attache à déterminer dans quelle mesure

\footnotetext{
${ }^{1}$ Pour une complète recension de la littérature concernant la théorie de la compensation et de la responsabilité, on se reportera à Fleurbaey et Maniquet (2006).
} 
l'origine familiale, repérée par le revenu du père, exerce un effet significatif sur le revenu des descendants en France.

Cet article cherche également à cerner la dynamique de cette inégalité des chances au cours des 20 dernières années. A cet égard, une énigme est apparue de la comparaison des résultats obtenus dans deux études précédentes.

L'approche usuelle consiste à estimer par une analyse de régression l'élasticité revenu intergénérationnelle. Cette élasticité mesure combien le revenu d'un individu augmente en réponse à une augmentation du revenu de ses parents. Lefranc et Trannoy (2005) ont procédé à la première estimation de cette élasticité à partir de données françaises. Les valeurs estimées des élasticités sont d'environ 0,4 pour les fils et de 0,3 pour les filles avec aucune tendance significative ni à la hausse ni à la baisse de 1964 à 1993. Cet article conclut donc à une permanence de l'inégalité des chances dans l'obtention du revenu salarial en France sur cette période. Cette approche de régression, pour courante qu'elle soit, comporte toutefois certaines limites. Elle revient à s'intéresser uniquement à l'espérance conditionnelle du revenu du descendant par rapport au revenu de l'ascendant. L'inégalité des chances n'est donc évaluée que sur la base de la comparaison d'une caractéristique de tendance centrale des distributions conditionnelles au milieu d'origine. Si la pente de la régression est non-significativement différente de zéro, on conclut à l'égalité des chances alors même que le milieu d'origine pourrait avoir un impact sur des moments supérieurs des distributions conditionnelles. Par exemple, le revenu parental pourrait avoir une influence sur la variance conditionnelle du revenu des descendants. Pour caractériser l'inégalité des chances, il convient donc d'étudier l'ensemble de la distribution de revenu conditionnelle au milieu d'origine.

Pour ce faire, on pourrait généraliser l'approche de régression en procédant à des régressions quantiles du revenu des descendants par rapport au revenu parental. C'est la démarche empruntée en partie par Dardanoni et alii (2005) pour des caractéristiques parentales différentes du revenu. Toutefois, cette démarche n'est pas entièrement générale : elle suppose une hypothèse paramétrique sur la relation entre quantile de revenu des descendants et caractéristiques du milieu d'origine. Une façon plus robuste de procéder est explorée par O'Neill et alii (2000) : dans un premier temps, ils estiment non- 
paramétriquement la distribution du revenu des descendants, conditionnelle au revenu des ascendants, pour toute valeur du revenu des ascendants; dans un second temps, ils comparent ces distributions conditionnelles pour certaines valeurs particulières du revenu des ascendants. La démarche statistique suivie par Lefranc, Pistolesi et Trannoy (2004) est un peu différente. Ils divisent la population en groupes de circonstances, les types pour Roemer. Puis ils comparent les distributions de revenu conditionnelles à ces types au moyen d'outils de dominance stochastique. en prêtant une attention particulière aux problèmes d'inférence statistique. Si deux distributions de revenu conditionnelles à des milieux d'origine distincts ne peuvent être classées à l'aide des instruments usuels de dominance stochastique, alors les auteurs admettent qu'il y a égalité des chances entre ces deux milieux. L'article cité ci-dessus conclut à une réduction de l'inégalité des chances chez les salariés lorsqu'on compare les destinées des fils selon la catégorie sociale de leur père.

Donc approximativement sur la même période d'étude, l'étude de régression conclut à une stabilité alors que l'étude de dominance retient une hypothèse de diminution de l'inégalité des chances. C'est l'un des enjeux de cet article que de savoir si ces différences de résultat sont le fruit de différences d'approche (régression d'une part, dominance d'autre part), de différences dans la variable, CSP ou revenu, permettant de définir les circonstances individuelles, ou même de différences de sources statistiques puisqu'elles n'ont pas été conduites sur les mêmes enquêtes, l'enquête FQP pour l'analyse de régression, l'enquête BDF pour l'analyse de dominance. En particulier, le revenu est un conditionnement beaucoup plus riche que la classe sociale et le conditionnement selon la CSP offre le désavantage de ne pas être invariant aux changements changements profonds de la structure socio-professionnelle : par exemple, la proportion des cadres augmente constamment, celle des agriculteurs chute.

Par ailleurs, l'un des inconvénients de l'approche de dominance est, par essence, qu'en se concentrant sur les inégalités entre différents milieux d'origine, elle laisse dans l'ombre l'inégalité des chances qui peut exister au sein de groupes d'origine imparfaitement homogènes. Or l'analyse de régression montre qu'une différence même ténue de revenu entre ascendants a un impact significatif sur le revenu des descendants. L'approche de dominance 
recourt nécessairement à une discrétisation du milieu d'origine qui est bien une variable continue, le revenu du père. Par là même, elle gomme les inégalités de circonstances intragroupes.

Pour tenter de réconcilier les résultats, approche discrète et approche continue de l'inégalité des chances doivent être menées de concert. Il semble naturel de travailler à partir de la même base de données, ici l'enquête FQP sur la période 1977-1993. L'annexe présente ces données et la façon dont nous les exploitons. Il convient en particulier de retenir que le revenu du père est un revenu prédit, exactement comme dans Lefranc et Trannoy (2005).

Cet article s'articule autour de deux parties. La première partie met en œuvre l'approche discrète de l'égalité des chances dans l'obtention du revenu et repère l'inégalité des chances à travers des tests de dominance. La méthodologie suivie est en tout point identique à celle retenue par Lefranc, Pistolesi et Trannoy (2004) et nous renvoyons le lecteur à cet article pour de plus amples précisions. Nous étudions en particulier la sensibilité des résultats à différents partitions de circonstances. La seconde partie utilise le caractère continu de la variable revenu pour proposer une analyse de décomposition de l'évolution de l'inégalité des chances à partir d'un indice d'inégalité, l'écart logarithmique moyenne (ELM) (mean logarithmic deviation). La méthodologie suivie est entièrement originale et montre qu'analyse de régression et décomposition de l'inégalité des chances selon l'ELM se combinent harmonieusement. Cela constitue une innovation méthodologique susceptible d'applications multiples. Cette analyse de décomposition permet surtout d'expliquer les différences de résultats obtenus dans les deux articles précédents ainsi que les différences de résultat de l'approche discrète suivant la partition retenue. La conclusion remet en perspective les résultats obtenus et les interprète au regard de l'éthique de l'égalité des chances.

\section{Approche discrète : égalité des chances et dominance stochastique}

La distinction classique entre critères d'équité et préordres sociaux traverse la théorie du choix social. Les premiers livrent des conditions qui définissent une situation équitable 
alors que les seconds fournissent des critères de classement des états sociaux. Nous nous contentons ici de proposer une définition d'une situation équitable à partir de concepts de dominance stochastique ${ }^{2}$ sans chercher à l'étendre à une possibilité de classement de situations inéquitables.

\subsection{Une définition à l'aide des concepts de dominance stochas- tique}

Le milieu social est représenté par une variable discrète notée $s, s \in S=\{1, \ldots, \bar{s}\}$. Le revenu du descendant est défini comme une variable continue $x$ sur $\mathbb{R}_{+}$. La fonction de répartition du revenu du descendant $x$ sachant $s$ est notée $F(x \mid s)$.

Le décideur social doit être en mesure de définir une relation de préférences sur $S$. Elle sera déterminée par la comparison des distributions conditionnelles, qui font partie de l'ensemble des distributions de probabilité sur la droite réelle positive. Les propriétés des préférences sur cet ensemble déterminent celles sur $S$. Nous faisons l'hypothèse que le décideur social agrée le critère de dominance stochastique au premier ordre $\left(\mathrm{SD}_{1}\right)$ et au second ordre $\left(\mathrm{SD}_{2}\right)$.

Définition L'origine sociale $s \mathrm{SD}_{1}$-domine l'origine sociale $s^{\prime}\left(s \succeq_{S D_{1}} s^{\prime}\right)$ ssi :

$$
F(x \mid s) \leq F\left(x \mid s^{\prime}\right) \forall x \in \mathbb{R}_{+}
$$

La dominance stricte $\left(s \succ_{S D_{1}} s^{\prime}\right)$ exige qu'il existe au moins un $x$ tel que $F(x \mid s)<$ $F\left(x \mid s^{\prime}\right)$.

L'application du critère de dominance stochastique d'ordre $13^{3}$ détermine un préordre sur $S$. Les milieux sociaux non-dominés sont définis pour la relation binaire $\succeq_{S D_{1}}$ comme

$$
P_{1}=\left\{s \in S \mid \nexists s^{\prime} \in S \text { tel que } s^{\prime} \succ_{S D_{1}} s\right\}
$$

Un préordre moins partiel correspondant à l'aversion au risque peut être défini quand

\footnotetext{
${ }^{2}$ Van de Gaer (1993) avait déjà proposé de s'appuyer sur des critères de dominance stochastique pour définir l'égalité des chances. Voir Lefranc et alii (2006) pour une justification plus approfondie des critères d'égalité des chances définis à partir des concepts de dominance stochastique.

${ }^{3}$ Martinez et alii (2001) le considèrent comme une propriété que devrait posséder toute mesure d'égalité des chances.
} 
les fonctions cumulées se coupent.

Definition L'origine sociale $s S D_{2}$-domine l'origine sociale $s^{\prime}\left(s \succeq_{S D_{2}} s^{\prime}\right)$ ssi :

$$
\int_{0}^{x} F(y \mid s) d y \leq \int_{0}^{x} F\left(y \mid s^{\prime}\right) d y \forall x \in \mathbb{R}_{+}
$$

Dans le cadre de la théorie de l'utilité espérée, la distribution dominante selon la $S D_{2}$ est préférée à la distribution dominée par tout agent qui éprouve de l'aversion au risque (sa fonction d'utilité est croissante et concave en $x$ ).

L'ensemble des milieux sociaux non-dominés pour $\succ_{S D_{2}}, P_{2}$, se définit d'une manière similaire à $P_{1}$. Étant donné que $S D_{2}$ est un critère moins partiel que $S D_{1}, P_{2} \subseteq P_{1}$. Nous définissons l'égalité des chances au sens faible comme une situation où il n'existe pas de milieux sociaux dominés pour la dominance stochastique à l'ordre 2. Cette définition de l'égalité des chances admet l'égalité des distributions conditionnelles comme cas particulier, c'est à dire le fait que

$$
F(x \mid s)=F\left(x \mid s^{\prime}\right) \forall x \in \mathbb{R}_{+} .
$$

Une telle situation décrit une situation d'égalité des chances au sens fort. C'est le concept retenu par Roemer (2004) et elle apparaît comme une définition concurrente de l'égalité des chances au sens faible. Une telle situation sera rarement observée en pratique. Une manière de rendre l'objectif plus accessible est bien de se contenter de l'égalité des chances au sens faible.

Notons pour finir que comparer des distributions conditionnelles centrées à leur moyenne peut avoir également un sens si l'on souhaite comparer les distributions conditionnelles à l'origine sociale du seul point de vue du risque, et indépendamment de leurs rendements respectifs. On utilisera alors le critère des courbes de Lorenz pour effectuer cette comparaison. D'une manière équivalente, le résultat de cette comparaison nous renseigne sur le caractère plus ou moins inégalitaire des distributions conditionnelles.

L'analyse des relations de dominance à partir de données d'enquête suppose de recourir à des techniques d'inférence statistique particulières qui relèvent d'une littérature théorique récente et en rapide évolution. Elle prend appui sur des résultats récents dus à Davidson 
et Duclos (2000) qui unifient les procédures de tests de dominance (pour n'importe quel ordre de dominance) et permettent, par l'absence d'hypothèse paramétrique particulière, d'aboutir à des conclusions statistiques plus solides. Nous mettons en œuvre la même méthodologie que dans Lefranc, Pistolesi et Trannoy (2004) et nous renvoyons donc le lecteur à cet article pour toute précision concernant l'inférence statistique.

Afin de mettre en œuvre ces critères d'égalité des chances, il est nécessaire de construire des types représentant l'ensemble des individus bénéficiant des mêmes circonstances.

\subsection{La partition en différentes circonstances}

On ne peut empêcher une part d'arbitraire dans la définition de ces groupes, puisqu'il s'agit de constituer une partition finie à partir d'une mesure continue de la circonstance, le revenu du père. Cet arbitraire aurait peu d'importance si on ne réalisait l'exercice que sur une seule vague d'enquête, c'est-à-dire si l'objet de la recherche ne présentait aucun aspect dynamique. Un de nos objectifs est au contraire de documenter l'évolution de l'inégalité des chances au cours du temps. Le choix d'une partition comparable au cours du temps est donc ici un enjeu important d'autant que la partition selon la CS retenue dans Lefranc, Pistolesi Trannoy (2004) souffre d'un problème de non-invariance longitudinale des groupes.

A cet égard, deux partitions s'imposent d'emblée. La première est une partition de nature purement ordinale, tandis que la seconde est de nature cardinale. Pour la première, deux individus appartiennent au même groupe si le revenu de leurs pères appartiennent au même quantile. Ainsi, les circonstances sont supposées comparables si le classement du revenu de leur père est le même. Cette première partition présente l'avantage que les groupes gardent une taille relative constante d'une période à l'autre. Toutefois, si la dispersion de la distribution du revenu des pères se modifie dans le temps, l'avantage ou le désavantage, en termes de niveau de revenu, associé à un quantile donné variera lui aussi. Appartenir au premier décile représente un handicap de circonstance, par rapport à la moyenne, d'autant plus faible que la dispersion des revenus est réduite chez les pères. C'est pour cette raison que l'on adopte également une seconde partition qui, cette foisci, considère que deux descendants ont les mêmes circonstances si le rapport du revenu de leur père au revenu moyen des pères appartient à un certain intervalle, fixe au cours 
du temps. Par construction, cette circonstance est invariante à un changement dans la dispersion des revenus des pères. Par contre, la taille relative des groupes peut varier selon la période. En cas de contraction de la distribution des revenus des pères, la taille des groupes proche de la médiane ou de la moyenne va augmenter au détriment des groupes en bas et en haut de l'échelle sociale. Ces deux types de classement entendent donc saisir deux dimensions différentes du revenu. Dans la première, ce qui importe pour le revenu futur du descendant c'est le rang du revenu du père, censé approcher des phénomènes de statut. Dans la seconde, c'est l'aisance monétaire qu'apporte le revenu que l'on cherche à cerner. Il se pourrait donc que l'évolution apparente de l'inégalité des chances diffère selon la partition retenue pour l'appréhender et dans la suite de l'article, nous adoptons ces deux approches.

Les valeurs des centiles servant à définir la partition ordinale des milieux d'origine sont données dans le tableau 1. Dans la définition de ces classes, on a cherché à obtenir des groupes d'origine sociale de taille suffisante pour mettre en œuvre les tests nonparamétriques de dominance stochastique. Entre la vague des descendants de 1977 et celle de 1993, on observe une réduction de la dispersion des salaires prédits des pères 4 : l'intervalle des valeurs observées, exprimé en multiples du salaire moyen, passe de [.377,3.1673] à $[.538,2.569]$.

La seconde partition est définie à partir de la valeur du salaire du père relativement au salaire moyen de la population des pères considérée : un groupe d'origine sociale est alors constitué des individus dont le père percevait un salaire (prédit) compris entre $x$ fois et $y$ fois le salaire moyen de l'année considérée. On maintient les bornes $x$ et $y$ inchangées quelque soit la vague d'enquête considéré£. Pour définir notre seconde partition de l'ensemble des milieux d'origine, on utilise pour bornes les valeurs du salaire (exprimées en multiples de la moyenne) servant à définir la partition relative ordinale de 1993. Pour cette vague, les deux partitions coïncident donc par construction. Les individus en 1977 ayant un salaire du père inférieur au seuil plancher ${ }^{6}$ de 1993 sont éliminés, ainsi que ceux ayant

\footnotetext{
${ }^{4}$ Pour une analyse de cette évolution, voir par exemple Goux et Maurin (1994).

${ }^{5}$ Il se peut qu'il n'ait pas une correspondance parfaite des bornes entre les deux dates, en raison du caractère discret des distributions, ce dont témoigne les légères différences de tranches dans le tableau 1 pour la définition cardinale.

${ }^{6}$ En écart à la moyenne.
} 
TAB. 1 - Définition des groupes d'origine sociale

\begin{tabular}{|c|c|c|c|c|c|c|c|}
\hline \multirow{3}{*}{$\begin{array}{c}\text { Classe } \\
\text { d'origine } \\
\text { sociale }\end{array}$} & \multicolumn{7}{|c|}{ définition ordinale } \\
\hline & \multirow[b]{2}{*}{ centiles } & \multicolumn{3}{|c|}{1977} & \multicolumn{3}{|c|}{1993} \\
\hline & & $x_{i n f}$ & $x_{m o y}$ & $x_{\text {sup }}$ & $x_{i n f}$ & $x_{\text {moy }}$ & $x_{\text {sup }}$ \\
\hline $\mathrm{C} 1$ & $1,15]$ & .377 & .499 & .555 & .538 & .635 & .687 \\
\hline $\mathrm{C} 2$ & {$[16,35]$} & .556 & .652 & .699 & .701 & .734 & .777 \\
\hline C3 & {$[36,55]$} & .704 & .775 & .839 & .781 & .833 & .867 \\
\hline $\mathrm{C} 4$ & {$[56,70]$} & .843 & .949 & 1.033 & .869 & .958 & 1.028 \\
\hline C5 & {$[71,85]$} & 1.034 & 1.223 & 1.443 & 1.031 & 1.153 & 1.367 \\
\hline \multirow[t]{4}{*}{ C6 } & {$[86,100]$} & 1.450 & 2.163 & 3.167 & 1.388 & 1.903 & 2.569 \\
\hline & \multicolumn{7}{|c|}{ définition cardinale } \\
\hline & & \multicolumn{3}{|c|}{1977} & \multicolumn{3}{|c|}{1993} \\
\hline & {$\left[x_{i n f}, x_{\text {sup }}\right]$} & \multicolumn{2}{|c|}{ centiles } & $x_{m o y}$ & \multicolumn{2}{|c|}{ centiles } & $x_{\text {moy }}$ \\
\hline C1 & {$[538,700]$} & \multicolumn{2}{|c|}{$[8,22]$} & .652 & \multicolumn{2}{|c|}{$1,15]$} & .635 \\
\hline $\mathrm{C} 2$ & {$[701,780]$} & \multicolumn{2}{|c|}{$[24,35]$} & .751 & \multicolumn{2}{|c|}{$[16,35]$} & .734 \\
\hline C3 & {$[781,868]$} & \multicolumn{2}{|c|}{$[38,43]$} & .822 & \multicolumn{2}{|c|}{$[36,55]$} & .833 \\
\hline $\mathrm{C} 4$ & {$[869,1.030]$} & \multicolumn{2}{|c|}{$[46,65]$} & .952 & \multicolumn{2}{|c|}{$[56,70]$} & .958 \\
\hline C5 & {$[1.031,1.387]$} & \multicolumn{2}{|c|}{$[67,84]$} & 1.145 & \multicolumn{2}{|c|}{$[71,85]$} & 1.153 \\
\hline C6 & {$[1.388,2.569]$} & \multicolumn{2}{|c|}{$[87,97]$} & 1.840 & \multicolumn{2}{|c|}{$[86,100]$} & 1.903 \\
\hline
\end{tabular}

Note : $x_{\text {inf }}, x_{\text {sup }}$ et $x_{\text {moy }}$ désignent respectivement les valeurs limites et la valeur moyenne des groupes d'origine sociale, exprimées en fraction du salaire moyen prédit des pères.

un salaire du père supérieur au salaire maximum de 1993. Au total, 442 observations ont été supprimées en 1977, soit environ 11\% de l'échantillon initial, $8 \%$ des individus du bas de la distribution, $3 \%$ des individus dans le haut. Le tableau 1 résume les différences entre les groupes suivant les deux approches.

La plupart des analyses de l'égalité des chances retiennent un conditionnement par la catégorie socio-professionnelle ou par le niveau d'éducation des ascendants. Le tableau 2 permet de comparer notre partition à celle généralement retenue dans la littérature7 7 . On constate que les groupes d'origine définis en fonction du revenu du père sont très proches des groupes que l'on pourrait constituer à partir de la seule information sur la CSP du père, et en particulier des groupes utilisés dans Lefranc et alii (2004). Les individus des deux premiers groupes sont quasiment tous enfants d'ouvriers. Ceux des groupes (C3) et (C4) sont pour un tiers des enfants de professions intermédiaire ou d'employés. Enfin les groupes (C5) (respectivement (C6)) sont constitués majoritairement de descendants de professions

\footnotetext{
${ }^{7}$ Le tableau est établi pour la partition cardinale. La partition ordinale ne change que très légèrement les résultats.
} 
TAB. 2 - Composition par CSP du père des groupes d'origine sociale

\begin{tabular}{|c|c|c|c|c|c|c|c|c|c|c|c|c|}
\hline \multirow[b]{3}{*}{ Cadres } & \multicolumn{6}{|c|}{1977} & \multicolumn{6}{|c|}{1993} \\
\hline & $\mathrm{C} 1$ & $\mathrm{C} 2$ & C3 & $\mathrm{C} 4$ & $\mathrm{C} 5$ & C6 & C1 & $\mathrm{C} 2$ & C3 & $\mathrm{C} 4$ & C5 & C6 \\
\hline & 0.0 & 0.0 & 0.0 & 0.0 & 1.7 & 80.9 & 0.0 & 0.0 & 0.0 & 0.0 & 0.0 & 74.6 \\
\hline Prof Interm & 0.0 & 0.0 & 0.0 & 26.6 & 85.0 & 19.1 & 0.0 & 0.0 & 0.0 & 39.9 & 84.2 & 23.7 \\
\hline Employés & 3.4 & 28.9 & 39.2 & 36.1 & 3.5 & 0.0 & 2.7 & 16.3 & 29.0 & 33.7 & 12.2 & 0.0 \\
\hline Ouvriers & 96.6 & 71.1 & 60.8 & 37.2 & 9.8 & 0.0 & 97.3 & 83.7 & 71.0 & 26.3 & 3.6 & 0.0 \\
\hline
\end{tabular}

intermédiaires (respectivement de cadres). En outre, sur la période, la composition des groupes change très peu, ce qui exclut toute explication des évolutions observées à partir d'une modification de la composition par CSP des groupes d'origine sociale retenus.

Le paragraphe suivant applique notre définition de l'égalité des chances, au conditionnement par la partition ordinale. Le paragraphe 2.4 exploite la partition cardinale.

\subsection{Approche ordinale : vers moins d'inégalité des chances?}

TAB. 3 - Tests de dominance stochastique - Approche ordinale

\begin{tabular}{|c|c|c|c|c|c|c|c|c|c|c|c|c|}
\hline \multirow[b]{3}{*}{$\mathrm{C} 1$} & \multicolumn{6}{|c|}{$\overline{1977}$} & \multicolumn{6}{|c|}{$\overline{1993}$} \\
\hline & C1 & $\mathrm{C} 2$ & C3 & $\mathrm{C} 4$ & C5 & C6 & C1 & $\mathrm{C} 2$ & C3 & $\mathrm{C} 4$ & C5 & C6 \\
\hline & - & $=$ & $<_{1}$ & $<_{1}$ & $<_{1}$ & $<_{1}$ & - & $=$ & $?$ & $<_{1}$ & $<_{1}$ & $<_{1}$ \\
\hline $\mathrm{C} 2$ & - & - & $<_{1}$ & $<_{1}$ & $<_{1}$ & $<_{1}$ & - & - & $=$ & $<_{1}$ & $<_{1}$ & $<_{1}$ \\
\hline C3 & - & - & - & $<_{1}$ & $<_{1}$ & $<_{1}$ & - & - & - & $<_{1}$ & $<_{1}$ & $<_{1}$ \\
\hline $\mathrm{C} 4$ & - & - & - & - & $?$ & $<_{1}$ & - & - & - & - & $=$ & $<_{1}$ \\
\hline C5 & - & - & - & - & - & $<_{1}$ & - & - & - & - & - & $?$ \\
\hline $\begin{array}{l}\text { Rev } \\
\text { et }\end{array}$ & $\begin{array}{l}\mathrm{ue} \\
\mathrm{col}\end{array}$ & iva & it $t$ & $\mathrm{DS}$ & ain. & & & $\begin{array}{l}\text { es } \\
\text { ion }\end{array}$ & tril & & & $\begin{array}{l}\text { sne } \\
\text { née }\end{array}$ \\
\hline
\end{tabular}

Les graphiques A et B de la figure 1 représentent les distributions de revenu des descendants, conditionnellement au milieu d'origine défini à l'aide de la partition ordinale des valeurs du revenu du père. En 1977, le classement des groupes correspond à la hiérarchie des salaires des ascendants. En effet, les distributions sont classées dans l'ordre de (C1) à (C6) de manière assez nette. Il est presque toujours préférable d'être issu d'un milieu 
social plus favorisé. Seules deux comparaisons, à l'œil nu, ne permettent pas de classer les groupes sociaux. La distribution des plus défavorisés (C1) coupe assez nettement celle du second groupe. En outre, la distribution du groupe 4 est très proche de celle du groupe 5 sauf dans les queues de distributions où les très hauts et les très bas salaires de ces milieux sociaux se distinguent. Les résultats des tests (tableau 3) confirment ces observations. Un ordre strict entre les groupes est observé sauf pour les groupes $(\mathrm{C} 1)$ et $(\mathrm{C} 2)$ qui sont statistiquement jugés égaux à $5 \%$, et pour les groupes $(\mathrm{C} 4)$ et $(\mathrm{C} 5)$ qui sont non-comparables. En 1977 les enfants les plus privilégiés se distinguent nettement du reste de la population. L'inégalité des chances liée a cette mesure relative apparaît forte.

En 1993, la figure 1 fournit un classement plus ambigu. D'une part, les milieux (C1), (C2) et (C3) sont beaucoup plus proches. D'autre part, la distance entre les milieux (C4), (C5) et (C6) parait plus ténue. A nouveau, les tests statistiques du tableau 3 confirment ces remarques, puisque les trois premiers milieux sociaux sont soit non-comparables, soit égaux entre eux. En outre, les tests concluent à l'égalité dans la comparaison de (C4) et $(\mathrm{C} 5)$ et à la non-dominance entre $(\mathrm{C} 5)$ et $(\mathrm{C} 6)$. Ce resserrement reflète les résultats déjà obtenus sur les données Budget de Familles sur la même période avec un conditionnement par rapport à la CSP dans Lefranc et alii (2004). En définitive si les tests concluent le plus souvent à l'inégalité des chances sur les deux vagues ( $86 \%$ des cas en 1977 , et $66 \%$ en 1993), ce chiffre en diminution sur la période, fait place dans $20 \%$ des cas à une égalité des chances forte et dans $13 \%$ des cas (2 comparaisons sur 15) à une non-comparabilité des distributions conditionnelles.

L'analyse précédente part du principe qu'un même rang dans la distribution de salaire du père engendre la même inégalité des chances en 1977 et en 1993. Or cette hypothèse est contestable. En effet, l'évolution de la distribution des salaires des pères au cours de la période n'est pas sans conséquence sur cette appréciation de l'inégalité des chances. La décroissance des inégalités salariales depuis les années 80 , conduit de fait à ce qu'un même rang dans la distribution se traduise par un écart au revenu moyen moins important. La section suivante reprend la même analyse en se focalisant sur les écarts au revenu moyen à l'aide de la partition cardinale. 


\section{A- 1977 - partition ordinale}

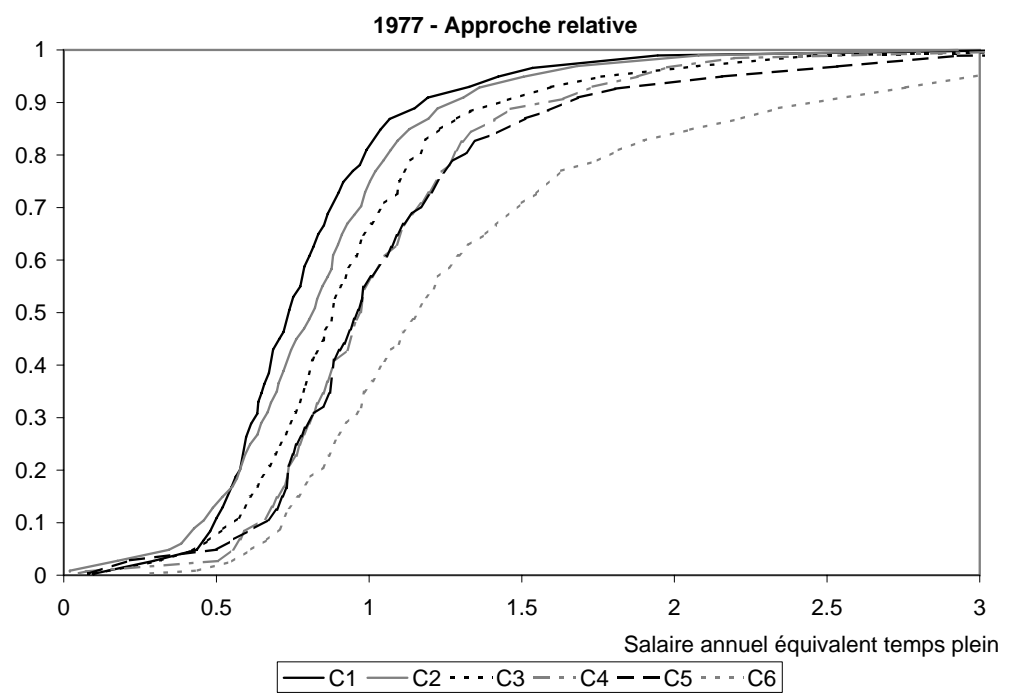

B- 1993 - partition ordinale et cardinale

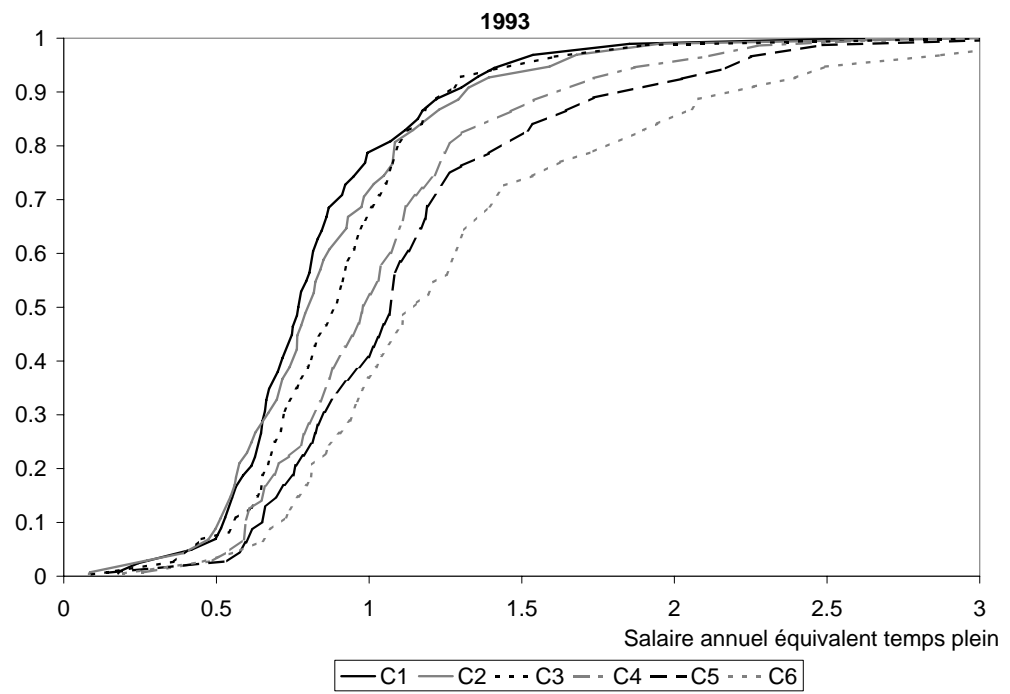

\section{C- 1977 - partition cardinale}

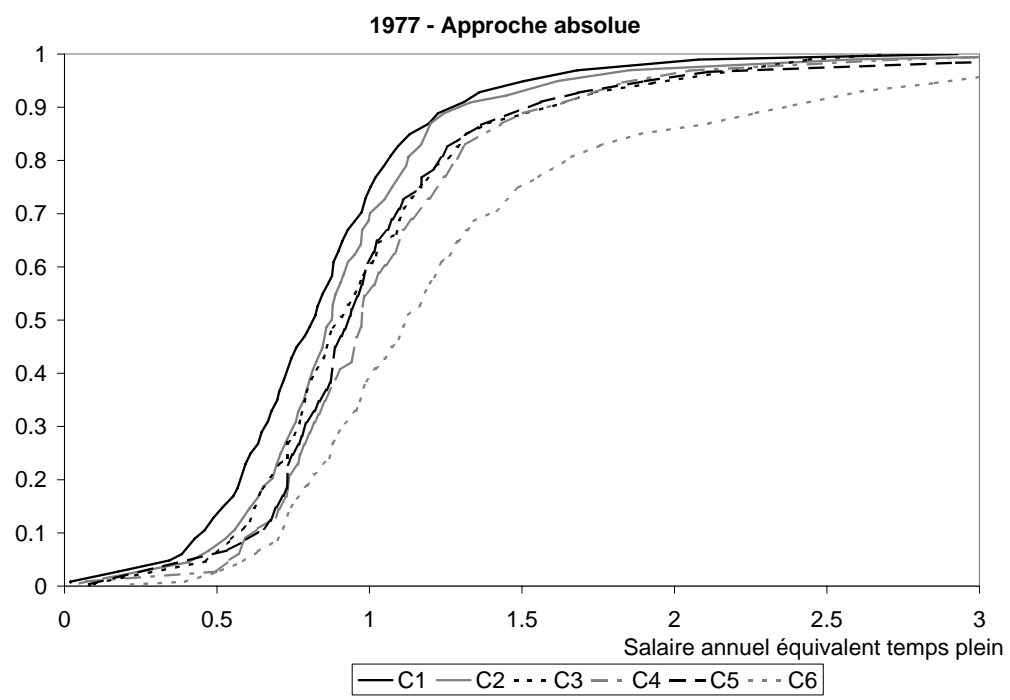

FIG. 1 - Distributions de revenu conditionnelles - mesure relative de l'inégalité des chances 
TAB. 4 - Tests de dominance stochastique - Approche cardinale

\begin{tabular}{|c|c|c|c|c|c|c|c|c|c|c|c|c|}
\hline & \multicolumn{6}{|c|}{1977} & \multicolumn{6}{|c|}{1993} \\
\hline & $\mathrm{C} 1$ & $\mathrm{C} 2$ & C3 & $\mathrm{C} 4$ & $\mathrm{C} 5$ & C6 & $\mathrm{C} 1$ & $\mathrm{C} 2$ & C3 & $\mathrm{C} 4$ & $\mathrm{C} 5$ & C6 \\
\hline $\mathrm{C} 1$ & - & $=$ & $<_{1}$ & $<_{1}$ & $<_{1}$ & $<_{1}$ & - & $=$ & $?$ & $<_{1}$ & $<_{1}$ & $<_{1}$ \\
\hline $\mathrm{C} 2$ & - & - & $=$ & $<_{1}$ & $=$ & $<_{1}$ & - & - & $=$ & $<_{1}$ & $<_{1}$ & $<_{1}$ \\
\hline C3 & - & - & - & $=$ & $=$ & $<_{1}$ & - & - & - & $<_{1}$ & $<_{1}$ & $<_{1}$ \\
\hline $\mathrm{C} 4$ & - & - & - & - & $=$ & $<_{1}$ & - & - & - & - & $=$ & $<_{1}$ \\
\hline C5 & - & - & - & - & - & $<_{1}$ & - & - & - & - & - & $?$ \\
\hline & $\overline{\mathrm{u}}$ & iva & th & ips & ein & Lect & & Les & istrib & & en & $\begin{array}{l}\text { sne } \\
\text { née }\end{array}$ \\
\hline
\end{tabular}

\subsection{Approche cardinale : une inégalité constante sur la période}

Les graphiques B et C de la figure1 1représentent les distributions de revenu des descendants, conditionnellement au milieu d'origine défini à l'aide de la partition cardinale des valeurs du revenu du père. Les résultats des tests de dominance apparaissent dans le tableau 4. Pour 1977, on observe un resserrement des distributions conditionnelles lorsqu'on passe de l'approche ordinale à l'approche cardinale. Alors que dans l'approche ordinale, la hiérarchie des groupes est assez transparente, avec l'approche cardinale, les distributions des milieux sociaux sont très proches sauf pour les groupes (C1) et (C6) qui s'écartent légèrement du reste de l'échantillon. Ces différences visuelles sont confirmées par les tests, puisque l'on conclut 6 fois (sur 15) à l'égalité avec l'approche cardinale au lieu d'une seule fois avec l'approche ordinale en 1977.

Par définition, pour 1993, approches ordinale et cardinale coïncident et les résultats sont identiques à ceux déjà discutés. On conclut à une inégalité assez forte sauf entre les 3 premiers groupes d'une part et les deux derniers groupes d'autre part. Avec l'approche cardinale, le nombre de fois où l'on observe l'égalité des chances passe de $40 \%$ à $20 \%$ de ces comparisons entre 1977 et 1993. Si bien qu'au lieu de conclure à un resserrement des distributions conditionnelles, cette fois-ci on trouve une certaine stabilité, voire une augmentation, de l'inégalité des chances. Au final, approche absolue et approche relative renvoient un message contradictoire. Les sections 3 et 4 expliquent cette contradiction. 
TAв. 5 - Tests de dominance de Lorenz

\begin{tabular}{|c|c|c|c|c|c|c|c|c|c|c|c|c|}
\hline & & & & & & & & & & & & \\
\hline & $\mathrm{C} 1$ & $\mathrm{C} 2$ & C3 & $\mathrm{C} 4$ & $\mathrm{C} 5$ & $\mathrm{C} 6$ & C1 & $\mathrm{C} 2$ & C3 & $\mathrm{C} 4$ & C5 & C6 \\
\hline $\mathrm{C} 1$ & - & $=$ & $=$ & $=$ & $?$ & $?$ & - & $=$ & $=$ & $=$ & $=$ & $?$ \\
\hline $\mathrm{C} 2$ & - & - & $=$ & $<$ & $?$ & ? & - & - & ? & $=$ & $=$ & $?$ \\
\hline C3 & - & - & - & $=$ & $=$ & ? & - & - & - & $=$ & ? & $?$ \\
\hline $\mathrm{C} 4$ & - & - & - & - & $=$ & $=$ & - & - & - & - & $=$ & $=$ \\
\hline C5 & - & - & - & - & - & $=$ & - & - & - & - & - & $=$ \\
\hline C6 & - & - & - & - & - & - & - & - & - & - & - & - \\
\hline $\begin{array}{l}\text { Rev } \\
\text { lign } \\
\text { don }\end{array}$ & & & & & & & & Les c & ourbe & $\mathrm{sde}$ & $\begin{array}{l}\text { oren } \\
\text { en } 1\end{array}$ & $\begin{array}{c}\text { en } \\
\text { gne }\end{array}$ \\
\hline
\end{tabular}

\subsection{Risque et rendement des distributions}

On représente le risque en centrant chaque distribution conditionnelle sur sa moyenne et les inégalités moyennes entre groupes s'en trouvent effacées. Les tests de dominance de Lorenz suivent une méthodologie identique aux tests de dominance stochastique. Le tableau 5 présente les résultats pour l'approche ordinal 8 . On constate que sur les deux vagues, une seule comparaison donne lieu à une relation de dominance. Le reste des tests conclue à l'égalité dans 2 cas sur 3 . Les différences de risques sont faibles, ce qui confirme les résultats de Lefranc, Pistolesi et Trannoy (2004) sur d'autres données françaises.
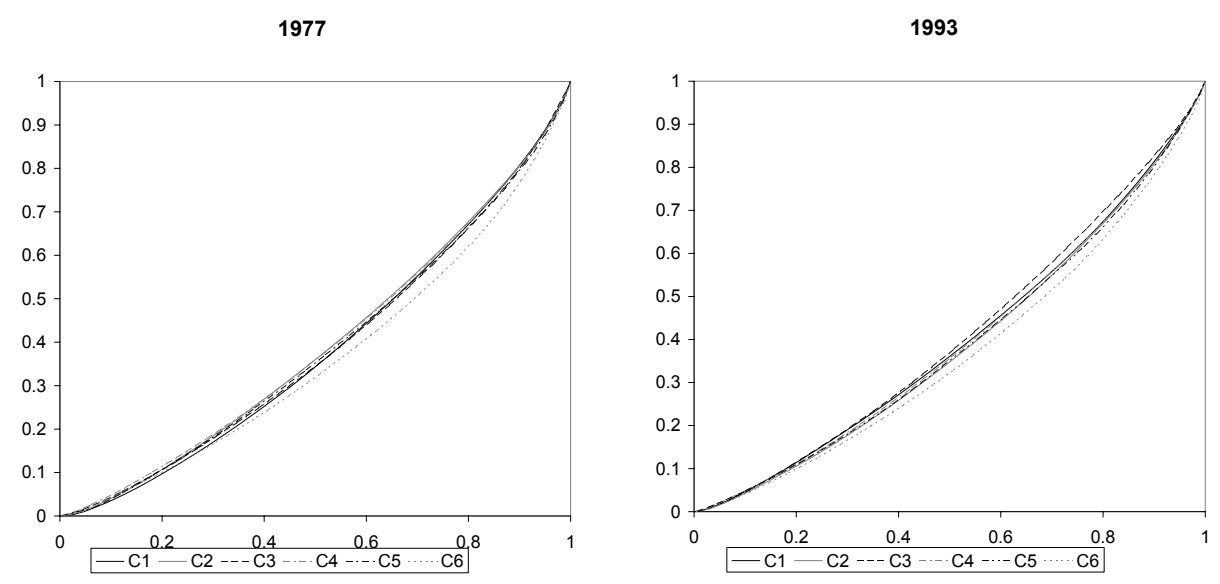

FIG. 2 - Courbes de Lorenz de distributions conditionnelles

${ }^{8}$ Des résultats équivalents sont obtenus pour l'approche cardinale. 
TAB. 6 - Evolution des revenus moyens conditionnels

\begin{tabular}{|c|c|c|c|c|}
\hline & \multicolumn{2}{|c|}{ Approche ordinale } & \multicolumn{2}{|c|}{ Approche cardinale } \\
\hline & 1977 & 1993 & 1977 & 1993 \\
\hline$(\mathrm{C} 6) /(\mathrm{C} 1)$ & 4.33 & 3.00 & 2.82 & 3.00 \\
\hline$(\mathrm{C} 6) /(\mathrm{C} 2)$ & 3.32 & 2.59 & 2.45 & 2.59 \\
\hline$(\mathrm{C} 6) /(\mathrm{C} 3)$ & 2.79 & 2.28 & 2.24 & 2.28 \\
\hline$(\mathrm{C} 6) /(\mathrm{C} 4)$ & 2.28 & 1.99 & 1.93 & 1.99 \\
\hline$(\mathrm{C} 6) /(\mathrm{C} 5)$ & 1.77 & 1.65 & 1.61 & 1.65 \\
\hline
\end{tabular}

En 1977, un descendant du groupe (C6) à un salaire moyen

plus de 4 fois supérieur au salaire moyen du groupe (C1).

Comme les risques sont peu différents, on peut étudier l'évolution des moyennes conditionnelles. Le tableau 6 indique que l'écart de revenus moyens entre groupes a diminué sur la période. Avec l'approche ordinale, le salaire moyen des individus les plus privilégiés est plus de 4 fois supérieur au salaire moyen des plus défavorisés en 1977. L'écart n'est plus que de 3 fois en 1993, soit une baisse d'un tiers. On peut remarquer que cette diminution confirme celle qui avait été observée en termes de revenu disponible sur les données Budget de Famille. Elle est d'une ampleur un peu plus forte que celle entre les fils de cadres et d'ouvriers, en revenu disponible avant impôts et transferts (cf Lefranc et alii (2004) tableau 4 page 70). La réduction de l'écart moyen est décroissante, le long de la distribution de revenu. Par contre, par définition, l'approche cardinale livre un verdict différent puisque les limites de tranches sont identiques : les ratios de revenus moyens n'évoluent pas sur la période. L'opposition de résultats entre l'approche ordinale et cardinale suggère une voie de recherche importante : il convient de cerner le rôle du degré d'inégalité de circonstances (qui est égale à l'inégalité des résultats de la génération précédente) et du processus de transmission de l'inégalité dans la constitution de l'inégalité des chances et son évolution. La section suivante développe cette analyse. 


\section{L'approche continue : des décompositions de l'inéga- lité des chances}

Nous développons maintenant une approche complémentaire de l'inégalité des chances. Celle-ci repose sur une représentation continue du processus de transmission intergénérationnelle des inégalités. Notre objectif est à la fois de mesurer l'inégalité des chances à l'aide d'un indice et de décomposer son évolution à l'aide de méthodes de régression. L'approche discrète, par essence, néglige l'inégalité des chances intra-groupes. L'approche continue permet d'apprécier l'importance de cette omission et d'en préciser la dynamique.

Combiner décomposition de l'inégalité et analyse de régression n'est pas chose aisée. L'approche usuelle utilise comme indice d'inégalité la variance des logarithmes, qui est bien appréciée des économètres mais peut entrer en contradiction avec les indications fournies par la courbe de Loren $4^{9}$ Pour rester cohérent avec le reste de l'article, nous choisissons un indice d'inégalité en accord avec le critère de Lorenz. Parmi les indices d'utilisation courante, la classe des indices d'inégalité de l'entropie présente l'avantage d'être additivement décomposable en une somme pondérée d'inégalités intra-groupes et une inégalité inter-groupes mesurée avec le même indice. L'ELM ${ }^{10}$ est le seul indice de cette classe à être basé sur une pondération selon les effectifs. Nous montrons qu'une analyse de décomposition peut être menée de concert avec l'analyse de régression lorsque l'on choisit l'ELM. Cette remarque, inédite dans la littérature sur l'inégalité des chances et, plus généralement, dans la littérature sur les inégalités, constitue l'innovation méthodologique de l'article. Nous commençons par l'exposer avant de la mettre en œuvre à partir des résultats de régression linéaire menée par Lefranc et Trannoy (2005)

\subsection{L'inégalité des chances mesurée par l'écart logarithmique moyen}

Soient $y_{i t}^{f}$ et $y_{i t}^{p}$ le revenu de l'individu $i$ et de son père à l'instant $t, \overline{y_{t}^{f}}, \bar{y} y_{t}^{p}$, leurs moyennes arithmétiques respectives, $a_{i t}$, l'âge du fils à l'instant $t$ et $\bar{a}_{t}$ l'âge moyen des fils

\footnotetext{
${ }^{9}$ Il se peut qu'il indique une baisse des inégalités alors même que la courbe de Lorenz indique une augmentation ou vice-versa (Ok et Foster (1999)).

${ }^{10}$ L'ELM est aussi le seul indice indépendant du pas de décomposition en utilisant la moyenne arithmétique comme revenu représentatif, voir Foster et Shneyrov (2000).
} 
en $t$. A partir des logarithmes des revenus centrés à leur moyenne arithmétique

$$
\widetilde{y}_{i t}^{f}=\log \frac{y_{i t}^{f}}{\overline{y_{t}^{f}}} \text { et } \widetilde{y}_{i t}^{p}=\log \frac{y_{i t}^{p}}{\overline{y_{t}^{p}}},
$$

Lefranc et Trannoy (2005) ont estimé la relation linéaire suivante à chaque vague

$$
\widetilde{y}_{i t}^{f}=\alpha_{t}+\beta_{t} \widetilde{y}_{i t}^{p}+\gamma_{t}\left(a_{i t}-\bar{a}_{t}\right)+\epsilon_{i t},
$$

où $\epsilon_{i t}$ est un résidu de moyenne nulle, non corrélé avec les explicatives et de variance finie. $\beta_{t}$ est l'élasticité revenu intergénérationnelle. On définit le log du revenu centré à l'âge moyen par

$$
\widehat{y}_{i t}^{f}=\alpha_{t}+\beta_{t} \widetilde{y}_{i t}^{p}+\epsilon_{i t}
$$

qui peut encore s'écrire

$$
\widehat{y}_{i t}^{f}=\widetilde{y}_{i t}^{f}-\gamma_{t}\left(a_{i t}-\bar{a}_{t}\right) .
$$

Choisissons pour mesurer l'inégalité chez les fils l'ELM soit

$$
I_{t}^{f}=\frac{1}{n} \sum_{i=1}^{n} \log \frac{\overline{y_{t}^{f}}}{y_{i t}^{f}}=-\frac{1}{n} \sum_{i=1}^{n} \widetilde{y}_{i t}^{f} .
$$

Il est immédiat de remarquer que

$$
I_{t}^{f}=-\frac{1}{n} \sum_{i=1}^{n} \widetilde{y}_{i t}^{f}-\frac{1}{n} \sum_{i=1}^{n} \gamma_{t}\left(a_{i t}-\bar{a}_{t}\right)=-\frac{1}{n} \sum_{i=1}^{n} \widehat{y}_{i t}^{f} .
$$

En utilisant la relation (4), on écrit

$$
I_{t}^{f}=-\alpha_{t}-\frac{1}{n} \beta_{t} \sum_{i=1}^{n} \widetilde{y}_{i t}^{p}-\frac{1}{n} \sum_{i=1}^{n} \epsilon_{i t}=-\alpha_{t}-\beta_{t} \frac{1}{n} \sum_{i=1}^{n} \widetilde{y}_{i t}^{p}
$$

d'où

$$
I_{t}^{f}=-\alpha_{t}+\beta_{t} I_{t}^{p}
$$

L'inégalité de revenu chez les fils, mesurée par l'ELM, s'écrit comme une fonction 
affine de l'inégalité des circonstances mesurée par l'ELM chez les pères. La constante $-\alpha_{t}$ s'interprète comme l'inégalité résiduelle s'il n'y avait pas d'inégalité des circonstances, c'est à dire, si tous les ascendants provenaient de la même famille. Dans ce cas, cette constante est égale à la différence des logarithmes des moyennes arithmétiques et géométriques.

Le modèle de régression linéaire, couplé avec l'utilisation de l'ELM comme indicateur d'inégalité, amène à une expression particulièrement simple de l'inégalité des chances, à savoir :

$$
I_{\text {oppt }}=\beta_{t} I_{t}^{p} .
$$

C'est l'inégalité qui subsisterait si le seul facteur de disparité entre les fils concernait le revenu de leur père. C'est le produit de l'élasticité intergénérationnelle de revenu et de l'inégalité des circonstances. La part que représente l'inégalité des chances dans l'inégalité des résultats est donc donnée par $\frac{\beta_{t} I_{t}^{p}}{I_{t}^{f}}$.11. Dans un régime stationnaire, elle est donc donnée $\operatorname{par} \beta_{t}$.

Notons pour mémoire que cette représentation particulièrement simple s'étend naturellement à un modèle où on ajoute une deuxième circonstance, par exemple le revenu de la mère. Avec des notations évidentes,

$$
\widetilde{y}_{i t}^{m}=\log \frac{y_{i t}^{m}}{y_{t}^{m}}
$$

on estimerait alors la relation

$$
\widetilde{y}_{i t}^{f}=-\alpha_{t}+\beta_{t}^{p} \widetilde{y}_{i t}^{p}+\beta_{t}^{m} \widetilde{y}_{i t}^{m}+\gamma_{t}\left(a_{i t}-\bar{a}_{t}\right)+\epsilon_{i t} .
$$

On obtiendrait alors

$$
I_{t}^{f}=-\alpha_{t}+\beta_{t}^{p} I_{t}^{p}+\beta_{t}^{m} I_{t}^{m}
$$

L'inégalité des chances est alors donnée par la formule suivante

\footnotetext{
${ }^{11}$ Il faut noter que dans l'application de cette formule à notre cas d'espèce, le revenu des pères est plutôt un revenu permanent alors que le revenu des fils est un revenu courant qui comporte une composante transitoire. L'inégalité du revenu des pères, tel que nous l'avons mesurée, a donc toutes les chances d'être plus petite que l'inégalité du revenu des fils. Par ailleurs, la valeur de l'élasticité tient compte de ce décalage puisque $\beta_{t}=\frac{\operatorname{cov}\left(y_{i t}^{f}, y_{i t}^{p}\right)}{\operatorname{var}\left(y_{i t}^{p}\right)}$
} 


$$
I_{\text {oppt }}=\beta_{t}^{p} I_{t}^{p}+\beta_{t}^{m} I_{t}^{m}
$$

Elle s'exprime donc comme une somme des inégalités de circonstances pondérée par les élasticités intergénérationnelles de revenu vis à vis du revenu du père et du revenu de la mère.

\section{Décomposition d'Oaxaca de l'inégalité des chances.}

Lorsqu'on considère l'évolution de l'inégalité des chances entre deux dates, l'écriture (8) se prête facilement à une décomposition à la Oaxaca-Blinder. L'inégalité des chances varie sous l'effet de deux facteurs, l'élasticité intergénérationnelle de revenu et l'inégalité des circonstances, c'est à dire, l'inégalité de revenu chez les pères. Il n'y a aucune raison de supposer que les deux forces évoluent dans le même sens. La première évalue la force de la transmission intergénérationnelle de la capacité économique tandis que la seconde traduit la disparité des conditions de départ. On obtient deux décompositions jumelles de l'évolution de l'inégalité des chances entre les dates $t$ et $t^{\prime}$,

$$
\Delta I_{\text {oppt }}=\Delta \beta_{t} I_{t}^{p}+\Delta I_{t}^{p} \beta_{t^{\prime}}=\Delta \beta_{t} I_{t^{\prime}}^{p}+\Delta I_{t}^{p} \beta_{t}
$$

avec $\Delta I_{\text {oppt }}=I_{\text {oppt }}-I_{\text {oppt }}$ et $\Delta \beta_{t}$ et $\Delta I_{t}^{p}$ les écarts correspondants entre $t$ et $t^{\prime}$.

Les premiers termes donnent l'impact du changement de l'élasticité intergénérationnelle appliquée à une inégalité des circonstances donnée, soit la valeur initiale soit la valeur terminale. Les seconds termes indiquent l'impact du changement de l'inégalité des circonstances appliquée à une élasticité intergénérationnelle donnée, soit la valeur initiale soit la valeur terminale.

Bien sûr, on peut accompagner cette analyse de décomposition de l'inégalité des chances par une décomposition de l'évolution de l'inégalité des revenus des fils

$$
\Delta I_{t}^{f}=\Delta \alpha_{t}+\Delta I_{\text {oppt }}
$$

Roemer interpréterait la variation de l'inégalité résiduelle comme la variation du résultat de l'effort. Nous nous défendons d'une telle interprétation car le terme résiduel peut 
encore recouvrir beaucoup de paramètres aux consonances très disparates : chance, effort, préférence et circonstances non-observées non-corrélées avec le revenu du père (Voir Lefranc et alii (2006)).

\section{Décomposition intra-groupe et inter-groupe}

Jusqu'ici nous avons raisonné sur la population totale. L'un des avantages de l'ELM est de se prêter à une analyse de décomposition intra-groupe et inter-groupes. Ici, nous considérons les deux partitions en 6 groupes (indicés par $j$ ) de la première partie. Cette décomposition permet de mesurer la perte d'information liée à ces partitions.

Elle peut se lire d'abord en coupe. En effet, pour chaque année, l'approche discrète conduit à ignorer les inégalités intra-groupe de milieu d'origine et l'inégalité des chances qu'elle engendre à la génération suivante. Nous savons qu'avec l'ELM, l'inégalité des revenus chez les descendants s'obtient comme une somme, pondérée par les poids démographiques des groupes, des inégalités intra-groupes et de l'inégalité inter-groupes.

$$
I_{t}^{f}=\sum_{j=1}^{6} \frac{n_{j}}{n} I_{j t}^{f}+\sum_{j=1}^{6} \frac{n_{j}}{n} \log \frac{\overline{y_{t}^{f}}}{\overline{y_{j t}^{f}}}=I_{W t}^{f}+I_{B t}^{f}
$$

où

$$
I_{j t}^{f}=\frac{1}{n_{j}} \sum_{i=1}^{n_{j}} \log \frac{\overline{y_{j t}^{f}}}{y_{i j t}^{f}}=-\frac{1}{n_{j}} \sum_{i=1}^{n_{j}} \widetilde{y}_{i t}^{f}
$$

avec des extensions de notations évidentes.

Mais d'autre part la formule de décomposition additive vaut également pour l'inégalité des circonstances, c'est à dire, l'inégalité chez les pères

$$
I_{t}^{p}=\sum_{j=1}^{6} \frac{n_{j}}{n} I_{j t}^{p}+\sum_{j=1}^{6} \frac{n_{j}}{n} \log \frac{\overline{y_{t}^{p}}}{\overline{y_{j t}^{p}}}=I_{W t}^{p}+I_{B t}^{p}
$$

et donc en vertu de (7) on peut également écrire que l'inégalité chez les fils est la somme du terme résiduel, de l'inégalité des chances intra-groupe et de l'inégalité des chances inter-groupe,

$$
I_{t}^{f}=-\alpha_{t}+\beta_{t} I_{W t}^{p}+\beta_{t} I_{B t}^{p}=-\alpha_{t}+I_{o p p W t}+I_{o p p B t}
$$


Il n'y a pas de relation évidente entre les deux écritures (10) et (12). Si les revenus moyens par groupe des pères sont égalisés, le troisième terme de (12) s'annule. Cela n'implique pas que l'inégalité inter-groupes chez les fils s'annule. De même si les revenus dans chaque groupe des pères sont égalisés, le deuxième terme de 112 s'annule. L'inégalité intra-groupe chez les fils ne s'annule pas pour autant.

Dans la première partie de cet article, nous nous sommes focalisés sur l'inégalité intergroupes $I_{B t}^{f}$ en l'identifiant à l'inégalité des chances, modulo le fait que nous nous intéressions à toute la fonction de répartition conditionnelle et pas seulement à la moyenne conditionnelle. Nous avons identifié l'inégalité intra-groupe $I_{j t}^{f}$ comme le degré de risque de gain au sein du groupe $j$. Il s'agit là au mieux d'une approximation, car en réalité il y a des différences de circonstances au sein de chaque groupe qui sont productrices de disparité au sein de chaque groupe. L'inégalité des chances intra-groupe ainsi négligée est égale à $\beta_{t} I_{W t}^{p}$.

Pour résumer, l'évolution de l'inégalité inter-groupe chez les descendants $\left(I_{B t}^{f}\right)$ doit être en phase avec ce que nous avons trouvé avec l'approche discrète. Par contre l'évolution de l'inégalité des chances $I_{o p p t}$ et de sa décomposition entre inégalité intra-groupe et intergroupe peut différer de l'évolution de $\left(I_{B t}^{f}\right)$. C'est un point que nous devrons vérifier dans notre application.

Maintenant, nous pouvons toujours affiner l'analyse en pratiquant une décomposition à la Oaxaca-Blinder de l'évolution de l'inégalité des chances intra-groupe et inter-groupe, c'est à dire,

$$
\begin{aligned}
\Delta I_{o p p W t} & =\Delta \beta_{t} I_{W t}^{p}+\Delta I_{W t}^{p} \beta_{t^{\prime}}=\Delta \beta_{t} I_{W t^{\prime}}^{p}+\Delta I_{W t}^{p} \beta_{t} \\
\Delta I_{\text {opp } B t} & =\Delta \beta_{t} I_{B t}^{p}+\Delta I_{B t}^{p} \beta_{t^{\prime}}=\Delta \beta_{t} I_{B t^{\prime}}^{p}+\Delta I_{B t}^{p} \beta_{t} .
\end{aligned}
$$

L'intérêt porte sur le terme de changement des inégalités de circonstances. On peut imaginer des situations où, par exemple, l'inégalité des circonstances diminue en intergroupe mais pas en intra-groupe. 


\subsection{Résultats : Une baisse de l'inégalité des chances due à la ré- duction de l'inégalité chez les pères}

Les tableaux 7 et 8 documentent l'étendue de l'inégalité totale et de l'inégalité des chances, et leur évolution entre 1977 et 1993. Le tableau 7 présente les résultats de l'estimation de l'équation de régression intergénérationnelle (3) et le tableau 8 calcule, à partir de cette estimation, une décomposition de l'inégalité totale entre inégalité des chances et inégalité résiduelle. Pour chacune des deux années considérées ici, deux points importants sont à noter. Premièrement, l'inégalité des chances représente au minimum un tiers de l'inégalité de résultat totale et donc le complémentaire, les différences d'effort ou de chance, compte pour au plus les deux tiers dans les différences de résultat entre individus Deuxièmement, seule une fraction de l'inégalité des circonstances $\left(I_{t}^{p}\right)$ est transmise à la génération suivante sous forme d'inégalité des chances $\left(I_{o p p} t\right)$ : cette fraction, de l'ordre d'un tiers, correspond justement à la valeur de l'élasticité intergénérationnelle de revenu $\beta$. Par ailleurs, l'évolution à l'œuvre entre 1977 et 1993 conduit à une réduction sensible de l'inégalité de résultat $\left(I_{t}^{f}\right)$, de l'ordre de $15 \%$. En outre, cette baisse de l'inégalité de résultat provient uniquement d'une réduction de l'inégalité des chances $I_{o p p}$. L'inégalité des chances baisse d'environ $30 \%$ au cours de la période. A contrario, le coefficient $\alpha$, qui mesure l'inégalité résiduelle, est remarquablement stable entre les deux dates et le tableau 7 indique que la différence entre les deux dates n'est pas statistiquement significative. Il convient donc d'identifier les facteurs qui ont pu contribuer à cette reduction de l'inégalité des chances.

Ici, les deux facteurs qui déterminent l'inégalité des chances ont évolué en sens opposé. On observe une forte réduction de l'inégalité de circonstances, de l'ordre de $40 \%$ (tableau 8 et une augmentation de l'élasticité intergénérationnelle qui passe d'environ 0.35 à 0.40 (tableau 7). L'intérêt de la décomposition d'Oaxaca-Blinder, rappelée dans l'équation (9) est de mesurer quelle aurait été l'évolution de l'inégalité des chances si seulement l'un de ces facteurs s'était modifié. Les résultats sont donnés dans le tableau 9. Si l'inégalité de

\footnotetext{
${ }^{12}$ On soulignera toutefois que l'inégalité totale est ici calculée à partir du revenu individuel annuel, qui incorpore une part importante de composantes transitoires. Si on retenait comme indicateur de résultat le revenu permanent, on obtiendrait alors une part plus importante de l'inégalité des chances dans l'inégalité de résultat.
} 
TAв. 7 - Régression de salaire intergénérationnelle

\begin{tabular}{lc}
\hline$\gamma$ & .0163 \\
$\beta_{77}$ & $(.0026)$ \\
& .3488 \\
$\beta_{93}$ & $(.0225)$ \\
& .4064 \\
$\alpha_{77}$ & $(.0359)$ \\
& -.0576 \\
$\alpha_{93}$ & $(.0107)$ \\
& -.0568 \\
Observations & $(.0123)$ \\
R-squared & 0.14754 \\
\hline \hline
\end{tabular}

Note : variable explicative : salaire annuel équivalent temps-plein. Le modèle estimé est celui de l'équation de régression intergénérationnelle (3). Le modèle est estimé à partir des échantillons principaux de 1977 et 1993 empilés.

TAB. 8 - Inégalité totale et inégalité des chances en 1977 et 1993

\begin{tabular}{lllllc}
\hline$t$ & $I_{t}^{f}$ & $I_{t}^{p}$ & $-\alpha_{t}$ & $I_{\text {opp } t}$ & $I_{\text {opp } t} / I_{t}^{f}$ \\
\hline $1977(1)$ & .1006 & .1233 & .0576 & .0430 & .4275 \\
$1993(2)$ & .0860 & .0716 & .0568 & .0291 & .3386 \\
$(1)-(2)$ & .0146 & .0516 & .0007 & .0139 & .0889 \\
\hline \hline
\end{tabular}


TAB. 9 - Décomposition Oaxaca-Blinder de l'évolution de l'inégalité des chances entre 1977 et 1993 - partition ordinale

\begin{tabular}{|c|c|c|c|c|c|}
\hline$t$ & $\Delta I_{o p p t}$ & $\Delta \beta_{t} I_{t}^{p}$ & $\beta_{t^{\prime}} \Delta I_{t}^{p}$ & $\frac{\Delta \beta_{t} I_{t}^{p}}{\Delta I_{o p p t}}$ & $\frac{\beta_{t^{\prime}} \Delta I_{t}^{p}}{\Delta I_{o p p t}}$ \\
\hline & & \multicolumn{4}{|c|}{ inégalité des chances totale $\left(I_{o p p} t\right)$} \\
\hline 1993 & 0.0139 & -0.0041 & 0.0180 & -0.2965 & 1.2965 \\
\hline 1977 & 0.0139 & -0.0071 & 0.0210 & -0.5106 & 1.5106 \\
\hline \multicolumn{6}{|c|}{ inégalité des chances inter-groupes $\left(I_{o p p B} t\right)$} \\
\hline 1993 & 0.0128 & -0.0039 & 0.0167 & -0.3043 & 1.3043 \\
\hline 1977 & 0.0128 & -0.0067 & 0.0195 & -0.5197 & 1.5197 \\
\hline \multicolumn{6}{|c|}{ inégalité des chances intra-groupes $\left(I_{o p p W} t\right)$} \\
\hline 1993 & 0.0011 & -0.0002 & 0.0013 & -0.2097 & 1.2097 \\
\hline 1977 & 0.0011 & -0.0004 & 0.0015 & -0.4094 & 1.4094 \\
\hline
\end{tabular}

circonstances était restée constante au cours de la période, on aurait observé une augmentation de l'inégalité des chances, sous l'effet de l'augmentation de $\beta$. Compte tenu du changement observé dans la valeur de $\beta$, l'augmentation de l'inégalité des chances aurait été de l'ordre de 10 à $15 \%$. A contrario, si $\beta$ était resté constant, on aurait dû constater une réduction d'environ $40 \%$ à $45 \%$ de l'inégalité des chances, au lieu des $30 \%$ effectifs. La réduction de l'inégalité des chances observée entre 1977 et 1993 provient donc intégralement (et au delà) d'une réduction de l'inégalité de circonstances et en aucun cas d'un affaiblissement de la force de transmission entre générations. Au contraire, ce lien tend à se renforcer, même s'il convient de rester prudent car les différences dans la valeur de $\beta$ entre 1977 et 1993 ne sont pas statistiquement significatives. En d'autres termes, l'égalisation partielle des revenus au sein de la génération des descendants, entre les deux dates, provient uniquement de l'égalisation des revenus au sein du groupe des pères, entre ces deux dates, et non pas d'un plus faible déterminisne social.

On peut affiner l'analyse en procédant à une décomposition de l'évolution des composantes de l'inégalité des chances (tableau 9), en reprenant la partition ordinale adoptée lors de la première partie. Les résultats obtenus concernant la contribution de l'élasticité intergénérationnelle et l'inégalité de circonstances à l'évolution de l'inégalité des chances se retrouvent lorsqu'on distingue inégalité des chances inter- et intra-groupes. En fait, in- 
égalités des chances inter- et intra-groupes évoluent de façon parallèle, sous l'effet (opposé) des mêmes causes : augmentation de l'élasticité et réduction de l'inégalité de circonstances.

Les résultats qui précèdent, obtenus à l'aide d'un modèle continu de transmission intergénérationnelle des inégalités, peuvent être reliés à ceux de la section 2 obtenus avec l'approche discrète. Ils permettent d'éclairer les conclusions contradictoires obtenues, selon qu'on adopte une partition ordinale ou cardinale de l'ensemble des milieux d'origine. On a vu que lorsqu'on adopte une partition ordinale, l'inégalité des chances diminue, alors que ce n'est pas le cas lorsqu'on retient une partition cardinale. La décomposition d'OaxacaBlinder permet d'expliquer ce hiatus. Par définition, la partition ordinale est affectée par la réduction de $I_{t}^{p}$ : celle-ci rend les différents groupes d'origine sociale plus proches les uns des autres, en termes de revenu. Ceteris paribus, on s'attend donc à un rapprochement des distributions de revenu conditionnelles à cette partition. Par contre, la partition cardinale n'est que très peu affectée par une réduction de $I_{t}^{p}$, car les bornes de groupes d'origine sociaux sont définies par la valeur du salaire, en multiples de la moyenne. Si on ignore les changements dans la distribution au sein de chacun des groupes, une baisse de $I_{t}^{p}$ doit donc laisser inchangée l'inégalité entre les groupes d'origine définis de façon cardinale. Toutes choses égales par ailleurs, on ne doit pas observer de rapprochement des distributions de revenu conditionnelles à cette partition. Par ailleurs, si la transmission des inégalités d'une génération à l'autre se renforce ( $\beta$ augmente), la partition cardinale doit mettre en évidence un écartement des distributions conditionnelles. C'est effectivement ce qu'on observe.

Le parallèle entre approche continue et approche discrète est toutefois imparfait. Comme on l'a déjà noté, l'approche discrète conduit en effet à effacer toute inégalité de circonstances au sein de chaque groupe d'origine sociale. Le tableau 10 procède à une décomposition de l'inégalité entre composante intra-groupe et composante inter-groupe et permet de quantifier l'ampleur de cet "oubli". L'oubli est en fait mineur. Raisonner à l'aide d'une partition discrète des milieux d'origine permet de saisir la plus grande partie des inégalités de circonstances : l'inégalité inter-groupes (celle qu'on observerait si les circonstances étaient égales au sein de chaque groupe) représente pratiquement $95 \%$ de l'inégalité des circonstances. Cette part a eu encore tendance à augmenter sur la période considérée ce 
TAB. 10 - Décomposition inter-groupe - intra-groupe de l'inégalité de circonstance en 1977 et 1993 - partition ordinale

\begin{tabular}{rrrr}
\hline$t$ & $I_{t}^{p}$ & $I_{B t}^{p}$ & $I_{W t}^{p}$ \\
\hline 1977 & 0.1233 & 0.1158 & 0.0075 \\
1993 & 0.0717 & 0.0678 & 0.0038 \\
\hline \hline
\end{tabular}

qui s'est traduit par une baisse plus soutenue de l'inégalité des circonstances intra-groupe que de celle inter-groupe (la première diminue de moitié, la seconde de $42 \%$ ).

En première approximation, l'approche discrète permet donc bien de saisir l'influence des circonstances extérieures à l'exercice de la responsabilité individuelle sur la formation du revenu. Pour se prononcer sur l'évolution au cours du temps de l'inégalité des chances et ses déterminants, elle suppose néanmoins d'établir la comparabilité au cours du temps des variables de conditionnement retenues.

\section{Conclusion}

La différence de résultats obtenus au moyen d'une approche de dominance, selon qu'on considère une définition ordinale (diminution de l'inégalité des chances) ou cardinale (relative stabilité) des circonstances s'éclaire à la lumière des résultats obtenus dans le cadre du modèle continu.

En optant pour une partition de la population des pères selon le niveau de revenu, on gomme tout effet possible d'un changement de l'inégalité des revenus dans cette génération sur la génération suivante. La seule source d’inégalité des chances réside alors dans la transmission de la capacité à générer de la réussite économique, qui est captée ici par l'élasticité intergénérationnelle de revenu. Or celle-ci est au mieux stable sur la période étudiée.

Par contre, en retenant une partition de la population des pères selon le rang, on s'autorise à répercuter l'effet d'une variation de l'inégalité des circonstances sur l'inégalité des revenus des descendants. La diminution de l'inégalité chez les pères, qui provoque bien une diminution de l'inégalité des chances dans l'approche continue, fait donc écho au résultat de baisse de l'inégalité des chances obtenue dans l'approche de dominance dans 
la version ordinale de la circonstance.

Les résultats obtenus dans cet article permettent également d'éclairer l'apparent hiatus entre les résultats obtenus dans Lefranc et alii (2004) qui trouvent une baisse de l'inégalité des chances lorsqu'on retient un conditionnement par rapport à la CS alors que Lefranc et Trannoy (2005) concluaient à une stabilité sur la base de la valeur de l'élasticité intergénérationnelle de revenu. Nous avons longtemps suspecté que la différence de conditionnement était à l'origine de la différence de résultat. Nous pouvons maintenant affirmer que la différence principale dans les résultats obtenus dans ces deux articles tenait simplement dans le fait qu'ils ne s'intéressaient pas aux même objets : l'inégalité des chances chez Lefranc et alii (2004) et la transmission de la capacité à engendrer du revenu chez Lefranc et Trannoy (2005). Cette différence d'objet nous a été masqué en raison de l'accent mis dans la littérature sur la mobilité intergénérationnelle sur la valeur de l'élasticité : c'est la donnée qui importe dans un état stationnaire. Le problème est que pour la France, la période qui nous occupe, 77-93, ne peut pas être considérée comme une période d'inégalité stationnaire, du moins chez les salariés. Cette période a connu une contraction de la disparité des salaires chez les pères.

Ces résultats débouchent sur un dilemme de politique économique, si on adopte l'éthique de l'égalité des chances à la Roemer. Réduire l'inégalité des résultats économiques chez les ascendants a deux effets. D'une part, cela s'interprète comme une réduction de l'inégalité des circonstances et cela réduit l'inégalité des chances pour la génération suivante. Cet effet est désiré par les tenants de l'éthique de la responsabilité. Mais d'autre part, en se plaçant du point de vue de la génération des ascendants, cette politique de réduction de l'inégalité des résultats s'est sans doute traduite par une moins grande rémunération de l'effort. Pour les philosophes de la responsabilité, une telle politique ne peut avoir que des effets ambigus : négatifs pour la génération présente, positifs pour la génération suivante. Ces philosophes soutiendraient par contre sans réserve une politique tendant à diminuer l'élasticité intergénérationnelle de revenu.

Maintenant, pour les tenants d'une égalisation des résultats, une telle politique présente un double dividende : une réduction de l'inégalité des résultats pour la période présente et une réduction de l'inégalité des chances pour la génération suivante qui toutes choses 
égales par ailleurs se transformera également en baisse de l'inégalité des résultats pour cette même génération. Une telle politique de baisse de l'inégalité des résultats pour une génération donnée risque donc d'avoir des répercussions sur l'inégalité dans la génération des enfants, mais également sur celle des petits-enfants toujours sous la clause ceteris paribus. A cet égard, il serait intéressant de prolonger l'étude en intégrant la vague 2003 de l'enquête FQP.

Sur un plan purement méthodologique, l'hypothèse de linéarité dans la transmission intergénérationnelle de revenu doit être considérée comme une première approximation. Cette élasticité peut varier le long de l'échelle des revenus, comme l'ont montré sur des données canadiennes l'étude de Corak et Heisz (1999). Sur nos données, des premiers tests indiquent que la valeur de l'élasticité diffère selon les groupes. Ces non-linéarités ${ }^{13}$ peuvent permettre de pratiquer une décomposition à la Oaxaca de l'évolution de l'inégalité intragroupe des revenus des descendants et de porter par là même un diagnostic plus fin sur l'évolution de l'inégalité des chances.

\section{Références}

Arneson, R. (1989). Equality and equal opportunity for welfare, Philisophical Studies $56: 77-93$.

Cohen, G. (1989). On the currency of egalitarian justice, Ethics 99 : 906-944.

Corak, M. and Heisz, A. (1999). The intergenerational earnings and income mobility of canadian men : evidence from longitudinal income tax data, Journal of Human Resources 84(3) : 504-533.

Dardanoni, V., Fields, G. S., Roemer, J. and Sanchez-Puerta, M. L. (2005). How demanding should equality of opportunity be, and how much have we achieved?, in S. Morgan, D. Grusky and G. Fields (eds), Mobility and Inequality : Frontiers of Reasearch in Sociology and Economics, Stanford University Press, Stanford.

Davidson, R. and Duclos, J.-Y. (2000). Statistical inference for stochastic dominance and for the measurement of poverty and inequality, Econometrica 68(6) : 1435-1464.

Dworkin, R. (1981). What is equality. part 1 : Equality of welfare, Philosophy and Public Affairs 10 : 185-246.

Fleurbaey, M. and Maniquet, F. (2006). Compensation and responsibility, in K. Arrow, A. Sen and K. Suzumura (eds), Handbook of Social Choice and Welfare, Vol. 2, Elsevier Science, New York.

\footnotetext{
${ }^{13}$ Voir O'Neill et alii (2000) pour une prise en compte de celles-ci dans une étude des ensembles d'opportunité.
} 
Foster, J. and Shneyerov, A. (2000). Path independent inequality measures, Journal of Economic Theory 91 : 199-222.

Goux, D. and Maurin, E. (1994). Education, expérience et salaire : tendances récentes et évolution de long terme, Économie et Prévision 116 : 155-178.

Lefranc, A. and Trannoy, A. (2005). Intergenerational earnings mobility in France : Is France more mobile than the US?, Annales d'économie et de statistique.

Lefranc, A., Pistolesi, N. and Trannoy, A. (2004). Le revenu selon l'origine sociale, Economie et statistique 371 : 49-82.

Lefranc, A., Pistolesi, N. and Trannoy, A. (2006). Equality of opportunity : Definitions and testable conditions, with an application to income in france, Mimeo THEMA.

Martinez, P., Schokkaert, E. and Van de Gaer, D. (2001). Three meanings of intergenerational mobility, Economica 68 : 519-537.

Ok, E. A. and Foster, J. E. (1999). Lorenz dominance and the variance of logarithms, Econometrica 67 : 901-908.

O'Neil, D., Sweetman, O. and Van de Gaer, D. (2000). Equality of opportunity and kernel density estimation : an application to intergenerational mobility, in T. Fomby and C. R. Hill (eds), Advances in Econometrics : Applying Kernel and Nonparametric Estimation to Economic Topics, Vol. 14, Elsevier Science, Oxford, pp. 259-274.

Osterbacka, E. (2001). Family background and economic status in Finland, Scandinavian Journal of Economics 103(3) : 467-484.

Roemer, J. (1998). Equality of Opportunity, Harvard University Press, Cambridge.

Roemer, J. (2004). Going beyond intergenerational income transition matrices, in M. Corak (ed.), Generational Income Mobility in North America and Europe, Cambridge University Press.

Van De Gaer, D. (1993). Equality of Opportunity and Investment in Human Capital, PhD thesis, K.U. Leuven.

\section{Annexe : Données utilisées}

L'idéal serait d'observer à la fois les revenus des individus et ceux de leurs ascendants. De telles données n'existent pas pour la France. Il est toutefois possible de surmonter cette limite en utilisant des enquêtes qui renseignent à la fois le revenu des individus et certaines caractéristiques socio-démographiques de leurs ascendants, corrélées au revenu (par exemple le niveau d'éducation ou la catégorie socio-professionnelle). A partir de ces caractéristiques on peut en effet prédire le revenu des ascendants. Pour cela, on utilise un échantillon auxiliaire représentatif de la population des ascendants, pour lequel on observe ces mêmes caractéristiques et les revenus. On estime une équation de gain en régressant le revenu sur les caractéristiques socio-démographiques disponibles dans l'enquête. Pour chaque individu, on prédit alors le revenu de ses ascendants à partir de cette équation et des caractéristiques déclarées de ses ascendant: ${ }^{14}$. On s'intéressera uniquement au revenu du père de l'individu. Les données proviennent de l'enquête FQP dont nous utilisons ici les vagues réalisées en 1964, 1977, et 1993. ${ }^{15}$

\footnotetext{
${ }^{14} \mathrm{On}$ pourrait s'inquiéter du fait que les déterminants les plus importants de la transmission d'une génération à une autre dans la capacité d'obtention d'un revenu passe par les inobservables. Osterbacka (2001) a toutefois montré que plus de la moitié de la corrélation intergénérationnelle est capturée par des variables individuelles observables.

${ }^{15}$ Toutes les vagues, à l'exception de 1993, reposent sur un échantillon stratifié de la population française en âge de travailler et les taux d'échantillonnage varient d'une strate à l'autre. Les résultats présentés sont
} 


\subsection{Sélection des échantillons}

Notre analyse repose sur deux séries d'échantillons : les échantillons principaux (ou échantillons de descendants) à partir desquels on mesure l'étendue de l'inégalité des chances ; des échantillons auxiliaires d'ascendants qui permettent de prédire les revenus des pères des individus des échantillons principaux.

Les échantillons de descendants proviennent des vagues 1977 et 1993 des enquêtes FQP. Pour chaque vague, nous nous restreignons aux individus âgés de 30 à 40 ans au moment de l'enquête et déclarant être chef de ménage ou conjoint du chef de ménage. Nous excluons les individus dont le rang de naissance est supérieur à 3 , afin de limiter l'intervalle d'âge admissible des pères des individus de notre échantillon des descendants (voir ci-dessous). Dans la mesure où nous n'observons les revenus d'activité que pour les salariés, nous excluons aussi les indépendants et les descendants d'indépendants. Enfin, les individus déclarant des revenus salariaux dont la valeur est inférieure à la moitié du salaire minimum sont exclus de notre échantillon.

Les échantillons auxiliaires proviennent des vagues 1964 et 1977 de l'enquête FQP. La vague 1964 (respectivement 1977) est utilisée pour prédire le revenu du père des individus de la vague 1977 (respectivement 1993). ${ }^{16}$ Nous nous restreignons aux hommes, chefs de ménage, salariés et père d'au moins un enfant au moment de l'enquête. Par ailleurs, nous ne retenons que les individus qui étaient âgés de 25 à 30 ans au moment de la naissance des individus des échantillons principaux ${ }^{17}$

\subsection{Principales variables}

L'analyse développée dans cet article se concentre sur deux variables : la variable de résultat, mesurée par le salaire équivalent temps-plein de l'individu et la variable de circonstance, définie par le salaire prédit de son père. Le salaire équivalent temps-plein est défini à partir du salaire annuel déclaré en tenant compte du nombre de mois travaillés à temps partiel et à temps complet au cours de la période de référence. Il constitue donc une mesure de la capacité de gain de l'individu, sur le marché du travail, plus que de son revenu effectif. D'autre part les revenus des descendants sont apurés des effets de l'âge. Dans toute l'analyse suivante ces revenus salariaux sont normalisés par le salaire moyen.

Le salaire du père de l'individu est prédit à partir de quatre caractéristiques observables, déclarées dans les échantillons principaux : le niveau d'éducation, la catégorie socio-professionnelle, l'appartenance au secteur public et le lieu de résidence ${ }^{18}$ du père. Le tableau 8 dans Lefranc et Trannoy (2005), donne les résultats des estimations servant à prédire le revenu du père. Devoir recourir au salaire prédit plutôt qu'au salaire effectivement perçu est en réalité moins gênant qu'il peut sembler. Le salaire effectif incorpore en effet des éléments transitoires qui sont peu reliés au revenu permanent qui caractérise le milieu d'origine d'un individu. Au contraire les différences de revenu liées à des différences d'éducation ou de catégorie socio-professionnelle sont plus durables et caractérisent mieux les circonstances auxquelles est soumis un individu. Rappelons pour mémoire que

donc pondérés par les fréquences d'échantillonnage.

${ }^{16}$ Les caractéristiques socio-démographiques du père déclarées par l'individu font référence au moment de la fin des études de l'individu.

${ }^{17} \mathrm{~L}$ 'âge moyen des pères à la naissance du premier enfant, dans nos échantillons, est proche de 27 ans. Comme les individus des échantillons principaux sont âgés de 30 à 40 ans à la date d'enquête, on se restreint donc, pour un échantillon auxiliaire de la vague d'enquête $v$ servant à prédire les revenus des ascendants d'individus issus de la vague d'enquête $v^{\prime}$, aux individus dont l'âge est compris dans l'intervalle $\left[30+25-v+v^{\prime}, 40+30-v+v^{\prime}\right]$.

${ }^{18} \mathrm{On}$ inclut une indicatrice de résidence en Île-de-France et une indicatrice de résidence en zone rurale. 
les résultats de Lefranc et Trannoy (2005) ont été obtenus avec un revenu prédit. Un conditionnement selon le revenu prédit est porteur de résultats différents de ceux obtenus avec un conditionnement selon la CS dans la mesure où l'explication du revenu prédit repose sur un grand nombre de variables significatives en plus de la CS. 